\title{
GROUPS WHICH ARE AN INFINITE CYCLIC EXTENSION OF A UNIQUE BASE GROUP
}

\author{
A. M. BRUNNER, JAMES MCCOOL and ALFRED PIETROWSKI
}

(Received 6 July 1976; revised 7 October 1976)

Let $G$ be a group which has exactly one normal subgroup $N$ with $G / N$ infinite cyclic; clearly such a group is an $H N N$ group with base $N$ and stable letter $t$, where $t$ induces an automorphism $\theta_{t}$ of $N$ under conjugation. We call such a group $G$ a unique base group with automorphism $\theta_{t}$ and base $N$.

Theorem 1 of this paper shows that the isomorphism problem for unique base groups is equivalent to a conjugacy problem in the outer automorphism group of the base group.

Any one-relator group with a finitely generated commutator subgroup is a two-generator unique base group, with base a finitely generated free group (Moldavanskiï (1967)). In Theorem 2 we establish some criteria for the isomorphism of such groups. We apply this, in Theorem 3, to generalise a result of Strasser (1959). We determine all two-generator one-relator groups which are an extension of a free group of rank two by an infinite cyclic group.

Let $w$ and $v$ be elements of a group $G$; then $w^{v}$ will denote the element $v^{-1} w v$, and $\langle w\rangle^{G}$ the least normal subgroup of $G$ which contains $w$.

\section{An isomorphism theorem}

We denote by $A(N)$ the automorphism group of the group $N$, and by $I(N)$ the group of inner automorphisms of $N$; the quotient $A(N) / I(N)$ is Out $(N)$ the outer automorphism group of $N$.

THEOREM 1. Let $C$ and $E$ be unique base groups with stable letters $c$ and $e$ respectively and the same base $N$. Then $C$ and $E$ are isomorphic if, and only if, $\theta_{c}$ is conjugate to $\theta_{e}^{ \pm 1}$ in Out $(N)$.

Proof. Let $\varphi$ be an isomorphism from $C$ to $E$. Then $\varphi$ induces an automorphism $\gamma$ of $N$. Also, since $C$ and $E$ have stable letters $c$ and $e$

Research partially supported by the C.S.I.R.O. of Australia and by a grant from the National Research Council of Canada. 
respectively, $c \varphi=e^{t} h$ where $\varepsilon= \pm 1$ and $h$ belongs to $N$. We show that $\gamma^{-1} \theta_{c} \gamma=\theta_{e}^{e} \tau$ where $\tau$ is the automorphism in $I(N)$ which corresponds to conjugation by $h$.

But, for each $n$ in $N$

$$
n\left(\gamma^{-1} \theta_{c} \gamma\right)=\left(c^{-1}\left(n \varphi^{-1}\right) c\right) \gamma=\left(e^{\varepsilon} h\right)^{-1} n\left(e^{\tau} h\right)=n \theta_{e}^{*} \tau .
$$

Hence $\theta_{c}$ is conjugate to $\theta_{e}^{\varepsilon}$ in $\operatorname{Out}(N)$.

Conversely, suppose $\theta_{c}$ and $\theta_{e}$ are conjugate in Out $(N)$. Then there is a $\tau$ in $I(N)$ and $\gamma$ in $A(N)$ such that $\gamma^{-1} \theta_{c} \gamma=\theta_{e}{ }_{e} \tau$. Let $\tau$ correspond to conjugation by $h$ in $N$.

Now $C=\left\langle c, N ;\right.$ rel $\left.N, c^{-1} n c=n \theta_{c}(n \in N)\right\rangle$, and $E=\left\langle e, N ;\right.$ rel $N, e^{-1} n e$ $\left.=n \theta_{e}(n \in N)\right\rangle$. Moreover, every element in $C$ can be uniquely expressed in the form $c^{r} n$, where $r$ is an integer and $n$ belongs to $N$. If $\varphi$ is the map from $C$ to $E$ defined by $\left(c^{r} n\right)_{\varphi}=\left(e^{r} h\right)^{r}(n \gamma)$ then it is easy to check, using the above descriptions of $C$ and $E$, that $\varphi$ is an isomorphism.

We will require the notion of Nielsen equivalence of presentations in the next section: two presentations of a group, given as factor groups $F / R$ and $F / S$, of a free group $F$ are said to be Nielsen equivalent if there is an automorphism $\alpha$ of $F$ with $R \alpha=S$.

Suppose $G$ is a unique base group with automorphism $\theta_{1}$ and base $N$. If $N^{\prime}$ denotes the derived group of $N$, it is clear that $G / N^{\prime}$ is also a unique base group with automorphism $\tilde{\theta}_{t}$ (induced by $\theta_{t}$ on $N / N^{\prime}$ ) and base $N / N^{\prime}$. When $G$ is two-generator, $G / N^{\prime}$ has one $T$-system of generating pairs (Brunner (1974), Theorem 2.4), and thus $G / N^{\prime}$ has one Nielsen class of two-generator presentations.

\section{Infinite cyclic extensions of free groups}

Let $\underset{X}{X}(r)$ stand for the class of two-generator one-relator groups which are an extension of a free group of finite rank $r$ by an infinite cyclic group.

As we shall see there is a close connection between the two-generator one-relator presentations of groups in $\underset{\underline{X}}{(}(r)$ and certain "diagonal" presentations:

$$
\Delta=\left\langle a, b ; a^{b^{\prime}}=u a^{\varepsilon} u^{\prime}\right\rangle,
$$

where $\varepsilon= \pm 1$, and $u, u^{\prime}$ belong to the (free) group generated by $a^{b}, \cdots, a^{b^{\prime-1}}$.

LEMMA 1. Let $G$ be a group with a diagonal presentation $\Delta$. Then $\langle a\rangle^{G}$ is a free group of rank $r$, and $G$ belongs to $\underset{\equiv}{X}(r)$.

Proof. Considerations such as those involved in Case 2 of Theorem 4.10 
of Magnus, Karrass, Solitar (1966), show that $a, a^{b}, \cdots, a^{b^{r-1}}$ generate freely a free subgroup of $\langle a\rangle^{G}$. But, since $a^{b^{\prime}}=u a^{\varepsilon} u^{\prime}$, it follows that $\langle a\rangle^{G}$ is actually generated by $a, a^{b}, \cdots, a^{b^{r-1}}$ and the assertion follows.

LEMMA 2. Any two-generator one-relator presentation of a group in $\underset{X}{X}(r)$ is Nielsen equivalent to a diagonal one.

This is a more general form of Lemma 1 of Strasser (1959), and its proof is exactly analogous to the one given there.

It follows that the isomorphism problem for two-generator one-relator presentations of groups in $\underset{=}{X}(r)$ reduces to the problem of determining when diagonal presentations define isomorphic groups.

Let $G$ be a group with a diagonal presentation $\Delta$; let $\sigma_{i}=\sigma_{i}(a, b)$ denote the exponent sum of $a^{-b^{\prime}} u a^{e} u^{\prime}$ on $a^{b^{i-1}}$ when $a^{-b^{r}} u a^{c} u^{\prime}$ is considered as a word in $a, a^{b}, \cdots, a^{b^{r}}$. Clearly $G / G^{\prime}$ is the direct product of an infinite cyclic group generated by $b G^{\prime}$, and (if $s \neq 0$ ) a cyclic group of order $s$ generated by $a G^{\prime}$; we call $s$ the torsion number of $G$.

LEMma 3. Let $G$ be a group with a diagonal presentation $\Delta$ and non-zero torsion number s. Then $G$ is a unique base group with base $H=\langle a\rangle^{G}$ and automorphism $\theta_{b}$.

Proof. Suppose $M$ is a normal subgroup of $G$ with $G / M$ infinite cyclic. Then $G^{\prime}$ is contained in $M$; hence $a$ belongs to $M$, since $a^{s}$ belongs to $G^{\prime}$ and $G / M$ has no elements of finite order. It follows that $H$ is contained in $M$. But $G / M \cong(G / H) /(M / H)$ is an infinite cyclic group, as is $G / H$; therefore $M=H$.

It is evident that $G$ is also a unique base group with base $H=\langle a\rangle^{G}$ and automorphism $\theta_{b^{-1}}$; moreover $\theta_{b}{ }^{-1}=\left(\theta_{b}\right)^{-1}$.

Now $H / H^{\prime}$ is a free abelian group, freely generated by $a H^{\prime}, a^{b} H^{\prime}, \cdots, a^{b^{r-1}} H^{\prime}$. Also, if $\tilde{\theta}_{b}$ denotes the automorphism of $H / H^{\prime}$ induced by $\theta_{b}$, then

$$
\left(a^{b^{\prime}} H^{\prime}\right) \tilde{\theta}_{b}=\left\{\begin{array}{l}
a^{b^{i+1}} H^{\prime} \quad \text { if } 0 \leqq i \leqq r-1 \\
a^{\sigma_{1}} a^{\sigma_{2} b} \cdots a^{\sigma_{r} b^{\prime}} H^{\prime} \text { if } i=r .
\end{array}\right.
$$

It follows that $\tilde{\theta}_{b}$, when considered as an element of $\operatorname{PSL}(r, Z)$, has the characteristic polynomial

$$
(-1)^{r}\left[\zeta^{r}-\sigma_{r} \zeta^{r-1}-\sigma_{r-1} \zeta^{r-2}-\cdots-\sigma_{1}\right]
$$

THEOREM 2. Let $C$ and $E$ be isomorphic groups in $\underset{=}{X}(r)$ with non-zero torsion number. If $(d, c)$ and $(f, e)$ are generating pairs of $\bar{C}$ and $E$, respectively, associated with diagonal presentations $\Delta$, then $\sigma_{k}(d, c)=\sigma_{k}\left(f, e^{E}\right)$ for $k=$ $1,2, \cdots, r$ where $\varepsilon= \pm 1$. 
Proof. Since $C$ and $E$ have non-zero torsion numbers they are unique base groups, by Lemma 3; as they are isomorphic we may assume they are given with base $N$ and automorphisms $\theta_{c}$ and $\theta_{e}$ respectively.

Now $N / N^{\prime}$ is a free abelian group of rank $r$, and clearly Out $\left(N / N^{\prime}\right) \cong$ $A\left(N / N^{\prime}\right) \cong P S L(r, Z)$. Thus, if $\tilde{\theta}_{c}$ and $\tilde{\theta}_{e}$ denote the automorphisms induced on $N / N^{\prime}$ then, by Theorem $1, \tilde{\theta}_{c}$ and $\bar{\theta}_{e}^{ \pm 1}$ are conjugate. Hence they have the same characteristic polynomial, and the result follows.

\section{Infinite cyclic extensions of free groups of rank two}

In this section we completely determine the one-relator presentations of

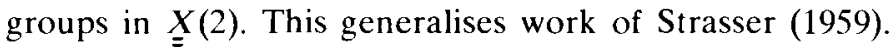

Let $P(k, \varepsilon)$ denote the presentation

$$
P(k, \varepsilon)=\left\langle x, y ; x^{y^{2}}=x^{\varepsilon} x^{k y}\right\rangle,
$$

where $\varepsilon= \pm 1$ and $k$ is an integer.

LEMMA 4. The group $C$ with presentation $P(0,1)$ is not isomorphic to the group $E$ having presentation $P(2,-1)$.

Proof. Let $V_{1}$ and $V_{2}$ denote the verbal subgroups of $C$ and $E$, respectively, generated by the third powers of the elements. Then, as can easily be checked, $C / V_{1}$ is the direct product of two cyclic groups of order 3 , while $E / V_{2}$ is the free two-generator group of exponent 3 (which is nonabelian of order 27 ).

THEOREM 3. Let $G$ be a group in $X(2)$ with torsion number $s$. Then any two-generator one-relator presentation of $G$ is Nielsen equivalent to one of $P(s, 1), P(2+s,-1)$ or $P(2-s,-1)$. When $s$ is positive these three groups are non-isomorphic; when $s=0$ the last two coincide.

Proof. By Lemma 2 a two-generator one-relator presentation is Nielsen equivalent to

$$
P=\left\langle x, y ; x^{y^{2}}=x^{k y} x^{c} x^{l y}\right\rangle \text {, where } \varepsilon= \pm 1 \text { and } k, l \text { are integers. }
$$

Replacing $y$ by $x^{k} y$ and leaving $x$ fixed, we see that $P$ is Nielsen equivalent to $P(n, \varepsilon)$ where $n=k+l$.

Suppose now that a group $C$ with presentation $P\left(n_{1}, \varepsilon_{1}\right)$ is isomorphic to a group $E$ with presentation $P\left(n_{2}, \varepsilon_{2}\right)$. Then, in particular, they have the same torsion number, so $n_{1}+\varepsilon_{1}-1= \pm\left(n_{2}+\varepsilon_{2}-1\right)$. We dispense first with the case that $n_{1}+\varepsilon_{1}-1=n_{2}+\varepsilon_{2}-1=0$ : when $\varepsilon_{1}=1$ we have $n_{1}=0$; when $\varepsilon_{1}=-1$ we have $n_{1}=2$. Moreover, by Lemma $4, P(0,1)$ and $P(2,-1)$ do not define isomorphic groups. 
Suppose now that $n_{1}+\varepsilon_{1}-1= \pm\left(n_{2}+\varepsilon_{2}-1\right) \neq 0$. By Theorem 2 there are two cases to be considered:

(a) $\varepsilon_{1}=\sigma_{1}(d, c)=\sigma_{1}(f, e)=\varepsilon_{2}$ and $n_{1}=\sigma_{2}(d, c)=\sigma_{2}(f, e)=n_{2}$;

(b) $\varepsilon_{1}=\sigma_{1}(d, c)=\sigma_{1}\left(f^{-1}, e\right)=\varepsilon_{2}$ and $n_{1}=\sigma_{2}(d, c)=\sigma_{2}\left(f^{-1}, e\right)=-n_{2} \varepsilon_{2}$.

Consider case (b). If $n_{1}+\varepsilon_{1}-1=n_{2}+\varepsilon_{2}-1$ then, as $\varepsilon_{1}=\varepsilon_{2}$, we have $n_{1}=n_{2}$. If $n_{1}+\varepsilon_{1}-1=-\left(n_{2}+\varepsilon_{2}-1\right)$, as $\varepsilon_{1}=\varepsilon_{2}$ and $n_{1}=-n_{2} \varepsilon_{2}$, in the one case $\varepsilon_{1}=\varepsilon_{2}=-1$ implies $n_{1}=n_{2}$, and in the other, $\varepsilon_{1}=\varepsilon_{2}=1$ implies $n_{1}=-n_{2}$.

We conclude that either $\varepsilon_{1}=\varepsilon_{2}$ and $n_{1}=n_{2}$, or $\varepsilon_{1}=\varepsilon_{2}=1$ and $n_{1}=-n_{2}$. This later possibility may be neglected because $P(n, 1)$ and $P(-n, 1)$ are Nielsen equivalent under the transformation which replaces $y$ by $y^{-1}$ and fixes $x$. Thus, for a given $s= \pm(n+\varepsilon-1)$ there are three solutions; $\varepsilon=1$ and $n=s, \varepsilon=-1$ and $n=2+s, \varepsilon=-1$ and $n=2-s$.

\section{References}

A. M. Brunner (1974), 'Transitivity-systems of certain one-relator groups', Proc. Second Internat. Conf. Theory of Groups, Canberra 1973 (Lecture Notes in Mathematics, 372, 131-140. Springer-Verlag, Berlin, Heidelberg, New York, 1974).

Wilhelm Magnus, Abraham Karrass, Donald Solitar (1966), Combinatorial Group Theory (Pure and Appl. Math. 13. Interscience [John Wiley \& Sons], New York, London, Sydney, 1966).

D. I. Moldavanskir (1967). 'Certain subgroups of groups with one defining relation', Siberian Math. J. 8, 1039-1048 (1968).

Elvira Strasser Rapaport (1959), 'Note on Nielsen transformations', Proc. Amer. Math. Soc. 10, 228-235.

Department of Mathematics,

York University,

Downsview,

Ontario, Canada.

Department of Mathematics,

University of Toronto,

Toronto,

Canada.

Department of Mathematics,

York University,

Downsview,

Ontario, Canada. 